ARTICLE

\title{
Early-onset autoimmunity associated with SOCS1 haploinsufficiency
}

Jérôme Hadjadj et al. ${ }^{\#}$

Autoimmunity can occur when a checkpoint of self-tolerance fails. The study of familial autoimmune diseases can reveal pathophysiological mechanisms involved in more common autoimmune diseases. Here, by whole-exome/genome sequencing we identify heterozygous, autosomal-dominant, germline loss-of-function mutations in the SOCS1 gene in ten patients from five unrelated families with early onset autoimmune manifestations. The intracellular protein SOCS1 is known to downregulate cytokine signaling by inhibiting the JAK-STAT pathway. Accordingly, patient-derived lymphocytes exhibit increased STAT activation in vitro in response to interferon- $\gamma, I L-2$ and IL- 4 that is reverted by the JAK1/JAK2 inhibitor ruxolitinib. This effect is associated with a series of in vitro and in vivo immune abnormalities consistent with lymphocyte hyperactivity. Hence, SOCS1 haploinsufficiency causes a dominantly inherited predisposition to early onset autoimmune diseases related to cytokine hypersensitivity of immune cells.

${ }^{\#}$ A list of authors and their affiliations appears at the end of the paper. 
T he immune system is tightly regulated by many central and peripheral checkpoints, in order to prevent reactivity to self-antigens. Their defects can lead to the expansion of self-reactive $\mathrm{B}$ and $\mathrm{T}$ lymphocytes, immune system overactivation, and thus autoimmune diseases ${ }^{1}$. Several monogenic defects leading to the breakdown of immune tolerance and autoimmunity have been identified, showing that a number of proteins are involved in non-redundant inhibitory checkpoints ${ }^{2}$. Moreover, the study of familial autoimmune diseases can reveal pathophysiological mechanisms also involved in more common autoimmune diseases ${ }^{2}$.

Cytokines, including interleukins and interferons (IFNs), participate in the development, differentiation, and effector functions of lymphoid and myeloid cells ${ }^{3}$. To do so, cytokines activate the Janus kinase (JAK)-STAT pathway that drives the transcription of cytokine-inducible genes ${ }^{3}$. Prolonged signaling (particularly by proinflammatory cytokines) can be harmful, requiring tight regulation of these pathways. The intracellular, inducible suppressors of cytokine signaling (SOCS) family of proteins is part of this negative-feedback system ${ }^{4}$. The SOCS family has eight members, all of which have a conserved central Src-homology 2 (SH2) domain (binding to JAKs' phosphotyrosine-containing sequences) and a short C-terminal SOCS box (causing the ubiquitination and proteasomal degradation of captured substrates) ${ }^{4}$. SOCS1 is the most potent member of the SOCS family, and is the primary downregulator of cytokines involved in immune responsesparticularly those that signal through the $\gamma \mathrm{c}$-chain (the IL-2 family) and interferons $s^{4,5}$. In addition to exerting ubiquitin ligase activity, SOCS1 directly inhibits JAK kinase activity through its kinase inhibitory region (KIR domain) ${ }^{5}$.

Here, using whole-exome/genome sequencing, we identify heterozygous germline SOCS1 mutations in ten patients from five unrelated families with early onset autoimmune manifestations and lymphoproliferation resulting in impaired SOCS1 function, increased STAT activation, and cytokine hypersensitivity of immune cells.

\section{Results}

Clinical features and immunological findings. We assessed ten patients from five unrelated kindreds presenting early onset autoimmune manifestations and SOCS1 mutations (see below) (Fig. 1a). The patients' main characteristics are summarized in Table 1 and cases are described in more detail in the Supplementary Notes. With the exception of one (E5), the first symptoms appeared during childhood (median (range) age: 7.5 years a

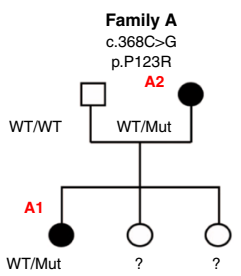

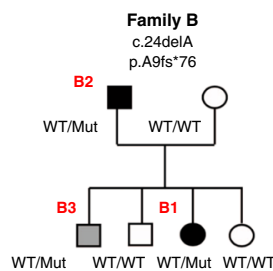
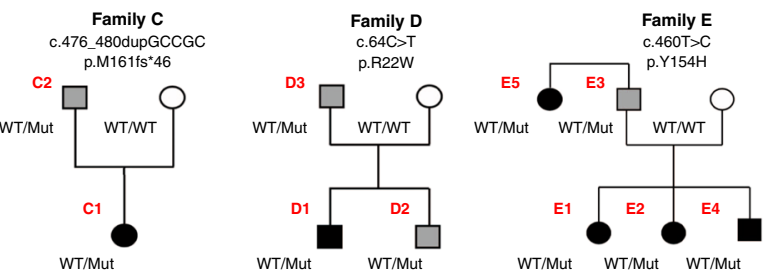

b

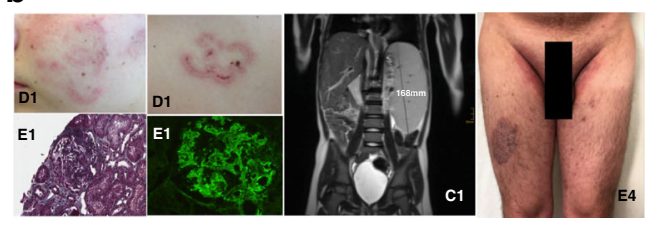

C

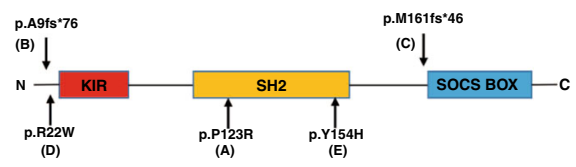

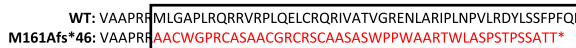

d e
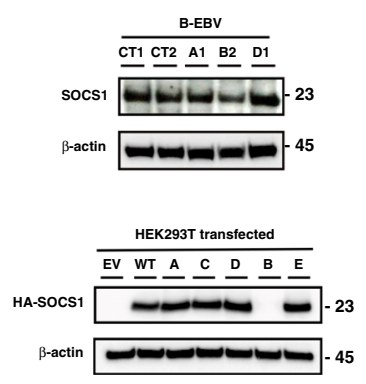

Fig. 1 Pedigrees and genetics of families with SOCS1 mutations. a Pedigrees of families with SOCS1 mutations. Squares: males; circles: females; black: affected mutation carriers; gray: unaffected mutation carriers. WT: wild-type SOCS1 allele. b Clinical manifestations in patients with SOCS1 mutations. Discoid lupus erythematosus (D1, upper left), active lupus nephritis (E1, lower left) with segmental cellular crescent associated with mesangial hypercellularity (Masson's trichrome $\times$ 400) and glomerular capillary wall and mesangial C1q deposition by immunofluorescence microscopy, abdominal MRI showing splenomegaly ( $\mathrm{C} 1$, middle), and plaque, intertrigous and guttata psoriasis (E4, right). c SOCS1 protein domains and locations of the mutations (upper panel, black arrows). The kinase inhibitory region (KIR) functions as a pseudosubstrate that can inhibit the tyrosine kinase activity of Janus kinase (JAK) proteins. The SRC-homology 2 (SH2) domain binds the activation loop of the JAK proteins' catalytic domain. The SOCS box recruits the ubiquitintransferase system and initiates the proteasomal degradation of JAK proteins. The SOCS1 M161Afs ${ }^{\star} 46$ mutant leads to a predicted $46-$ residue neopeptide in the SOCS box domain three amino acids shorter than the wild-type protein (lower panel). d Top panel: position of the P123 and Y154 in human SOCS1 within a 3D model of the JAK1/SOCS1 complex. Bottom panel: a model of the human SOCS1's SH2 domain. The two mutated amino acids (P123R and $\mathrm{Y} 154 \mathrm{H}$ ) are highlighted in the phosphotyrosine peptide binding groove (the $\mathrm{pY}$ and $\mathrm{pY}+3$ pockets). The possible location of a phosphotyrosine peptide is shown in purple. e SOCS1 protein expression in patient-derived cells and transfected cells. Top: Western blot (WB) analysis of lysates from Epstein-Barrvirus (EBV)-tranformed B cells from patients A1, B2, and D1 and from two healthy controls (CT1 and CT2), following incubation with anti-SOCS1 antibodies (upper panel) or anti-actin antibodies as a loading control (lower panel). Bottom: HEK293T cells transiently transfected with an empty vector (EV), a vector coding for hemagglutinin (HA)-tagged wild-type (WT) SOCS1 protein, or vectors coding for the five HA-tagged mutant SOCS1 proteins. Lysates were incubated with anti-HA antibodies (upper panel) or anti-actin antibodies as a loading control (lower panel). Data are representative of two independent experiments. 


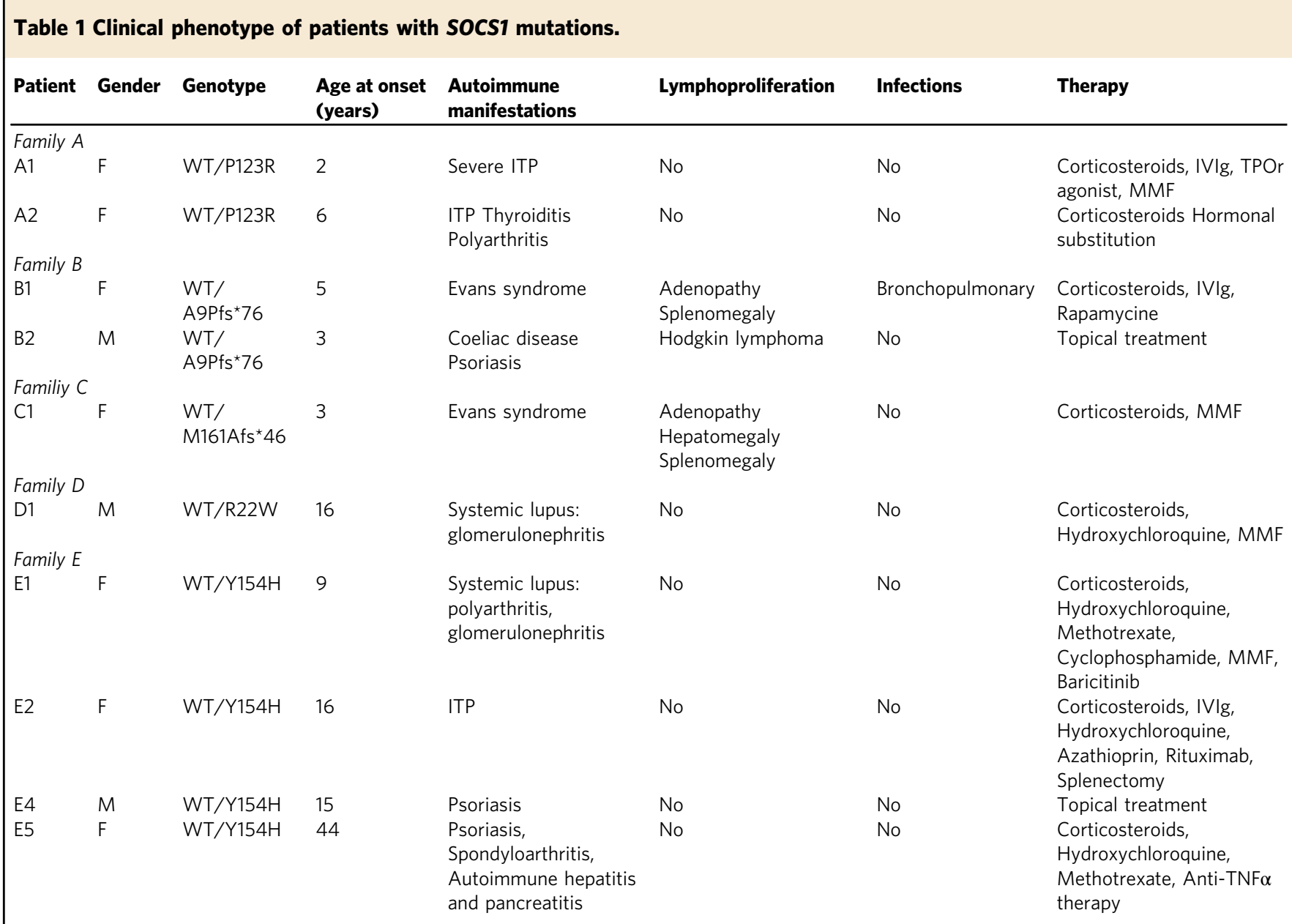

F female, M male, WT wild-type, ITP immune thrombocytopenia, IVIg intravenous immunoglobulin, TPOr TPOr receptor, MMF mycophenolate mofetil, TNF tumor necrosis factor.

(2-44)) in all patients. Five patients (A1, A2, B1, C1, and E2) had autoimmune cytopenia, four patients (A2, B2, E4, and E5) had organ-specific autoimmunity (thyroiditis, coeliac disease, psoriasis, spondyloarthritis, and hepatitis), and two patients (D1 and E1) met the diagnostic criteria for systemic lupus erythematosus (SLE) with skin (discoid lupus) and kidney (lupus glomerulonephritis) involvement (Fig. 1b). Two patients (B1, and C1) had benign lymphoproliferation (lymphadenopathy and/or hepatosplenomegaly (Fig. 1b)), whereas one (B2) developed Hodgkin lymphoma. None had infectious complications, with the exception of recurrent bronchopulmonary infections in B1.

Immunological investigations were performed on peripheral blood mononuclear cell samples from all patients (Supplementary Table 1). The marginal zone and/or switched memory B cell counts were both low in seven patients (Supplementary Fig. 1A and Supplementary Table 1), whereas IgG levels were normal. Elevated CD21 low CD38 low B-cell counts (that may include autoreactive $\mathrm{B}$ cells) were observed in seven out of eight patients (Supplementary Fig. 1B). Eight of the nine patients tested had autoantibodies, including antinuclear autoantibodies $(n=5)$, anti-DNA antibodies $(n=3)$, antinuclear antigen antibodies (anti-SSA, anti-RNP, anti-SCL, $n=5$ ), and a positive Coombs test $(n=3)$. It is noteworthy that plasma $\mathrm{B}$-cell activating factor (BAFF) levels were elevated in five of the eight patients tested (Supplementary Table 1). Although the T and NK cell counts were variable, no cell subsets specific abnormalities were found.
Identification of SOCS1 mutations. Whole-exome or genome sequencing was performed on whole blood DNA samples from six patients (A1, A2, B1, C1, D1, and E1). Heterozygous germline SOCS1 mutations were identified in all six patients, including three missense mutations in families A (c.368 C > G, p.P123R), D (c.64 C $>$ T, p.R22W) and E (c.460 T > C, p.Y154H), respectively, and two frameshift mutations in families B (c.24delA, p.A9fs*76) and C (c.476_480dupGCCGC, pM161fs*46) (Fig. 1a, Supplementary Fig. 2A). All SOCS1 variants were predicted to be deleterious, with a high CADD (Combined Annotation-Dependent Depletion) score above the cutoff score of 11.63 indicating mutational significance ${ }^{6}$, and were absent or very rare in public gene mutation databases (Supplementary Fig. 2b). Two mutations (in families B and D) were located at the $5^{\prime}$ end of the kinase inhibitory region, two (in families $\mathrm{A}$ and $\mathrm{E}$ ) were located in the $\mathrm{SH} 2$ domain, and one (in family $\mathrm{C}$ ) was located at the $5^{\prime}$ end of the SOCS-box domain (Fig. 1c). Three distinct missense variants (in families A, D, and E) affected phylogenetically conserved amino acids (Supplementary Fig. 2C). It is noteworthy that residues P123 and Y154 were previously reported as a site of somatic mutations in B cell lymphomas ${ }^{7,8}$. An analysis of the SOCS1 three-dimensional (3D) protein structure showed that P123 and Y154 are located within the groove (Fig. 1d) that binds phosphorylated JAK peptides with high affinity ${ }^{5}$. 3D structure information was not available for R22, belonging to a disordered segment. In view of its position (upstream of the kinase inhibitory region), this mutation could perhaps perturb binding to JAK peptides. Details of the SOCS1 mutants' $3 \mathrm{D}$ modeling and 
analysis are given in the Supplementary Notes and in Supplementary Fig. 3-4. Mutations were then identified in four additional patients (B2, E2, E4, and E5) as well as five apparently healthy carriers. The familial segregation thus indicated autosomaldominant inheritance with incomplete clinical penetrance (Fig. 1a).

The impact of SOCS1 mutations on protein expression was analyzed using patient-derived EBV-transformed B cell lines and HEK293T cells transfected with the various SOCS1 alleles. Ectopic expression of the p.A9Pfs*76 mutation (family B) did not yield detectable protein levels (Fig. 1e bottom and Supplementary Fig. 5) while lower expression was detected in the corresponding EBV-B mutant cell line, indicating haploinsufficiency (Fig. 1e top). The frameshift mutation in family $\mathrm{C}$ yielded a detectable protein containing a predicted 46-residues neopeptide within the SOCS box domain and a predicted premature stop codon removing the last three amino acid residues (Fig. 1c-e). Other mutants were normally expressed in both EBV-B cells and HEK293 T cells.
Consequence of SOCS1 mutations on STAT pathway activation. To examine the impact of the SOCS1 mutations on the regulation of the STAT pathways in patients' lymphocytes, EBV-B cells from A1, B2, and D1 were stimulated with various cytokines and STAT phosphorylation was determined as a surrogate of activation $^{3}$. Of note, constitutive phosphorylation of the STAT1, STAT5 and STAT6 proteins was not detected. However, levels of IFN- $\gamma$-induced STAT1 phosphorylation, IL-2-induced STAT5 phosphorylation, and IL-4-induced STAT6 phosphorylation were higher in patients' cells than in control cells (Fig. 2a, b and Supplementary Fig. 6). Consistently, we observed elevated nuclear translocation of P-STAT1 and P-STAT5 upon stimulation with IFN- $\gamma$ and IL-2, respectively (Fig. 2c). Furthermore, expression of the IFN- $\gamma$ inducible CXCL9 and CXCL10 genes and the IL-2inducible CISH and PIM1 genes was upregulated in patient cells following cytokine stimulation (Fig. 2d). Accordingly, no baseline hyperactivity was detected in patients' primary monocytes but IFN$\gamma$-induced STAT1 phosphorylation was also moderately but sig-
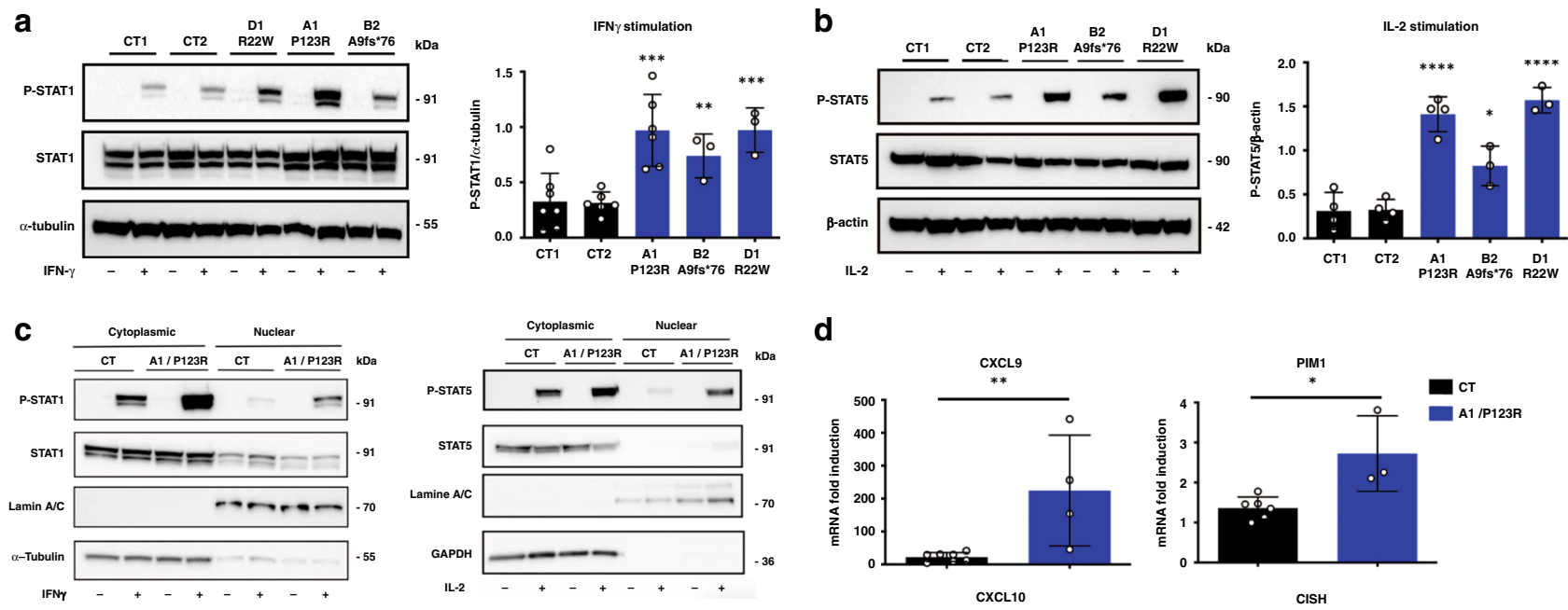

d
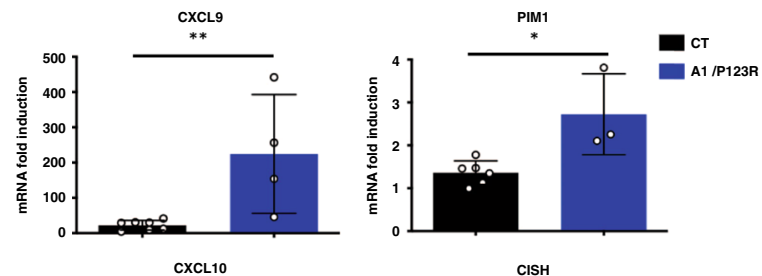

e
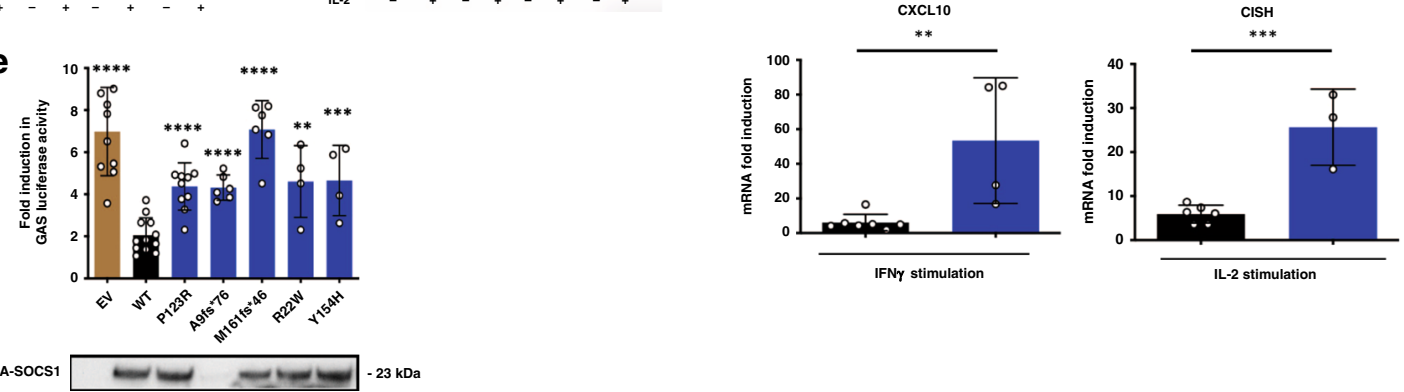

Fig. 2 SOCS1 mutations result in uncontrolled STAT pathways activation. a, b Left: Western blots (WB) of patients (A1, B2, and D1) and healthy controls (CT) derived EBV-B cells stimulated with IFN- $\gamma\left(10^{3} \mathrm{IU} / \mathrm{ml}\right.$ for $\left.1 \mathrm{~h}\right)(\mathbf{a})$ or $\mathrm{IL}-2\left(10^{4} \mathrm{IU} / \mathrm{ml}\right.$ for $\left.2 \mathrm{~h}\right)$ (b). Lysates were incubated with an antibody against tyrosine-phosphorylated STAT (P-STAT) or against total STAT, as indicated. Right: densitometric quantification of the phospho-STAT/ $\alpha$-tubulin or $\beta$-actin ratio upon stimulation. $\mathbf{a}, \mathbf{b}$ Data are representative of $n=3$ (patients B2 and D1), $n=4$ (A1, IL-2 stimulation), and $n=6$ (A1, IFN $\gamma$ stimulation) independent experiments. Statistics (versus CT2): IFN $\gamma$ stimulation, A1 $p=0.0008, \mathrm{~B} 1 p=0.0029$, D1 $p=0.0002 ; \mathrm{IL}-2$ stimulation, A1 $p<0.0001, \mathrm{~B} 1 p=0.0118, \mathrm{D} 1$ $p<0.0001$. c The nuclear and cytoplasmic fractions of EBV-B cells from a control (CT) and from patient A1 after stimulation with IFN- $\gamma$ for $1 \mathrm{~h}$ (left) or with IL-2 for $2 \mathrm{~h}$ (right) were tested by WB for the presence of P-STAT1 and P-STAT5, respectively. Anti-lamin A/C and anti- $\alpha$-tubulin antibodies were used to normalize the amount of nuclear and cytoplasmic proteins. Data are representative of two independent experiments. d Real-time quantitative RT-PCR assays of CXCL9 and CXCL10 expression $6 \mathrm{~h}$ after stimulation with IFN- $\gamma$ (left), and assays of CISH and PIM1 expression $6 \mathrm{~h}$ after stimulation with IL-2 (right) in EBV-B cells from a CT and from patient A1. Results represent the fold-increased expression between stimulated and unstimulated states and are normalized to endogeneous GAPDH. Data are representative of $n=3$ (IL-2 stimulation) and $n=4$ (IFN $\gamma$ stimulation) independent experiments performed in triplicate. Statistics: IFN $\gamma$ stimulation, CXCL9 $p=0.0094$, CXCL10 $p=0.0063$; IL-2 stimulation, PIM1 $p=0.0105$, CISH $p=0.0008$. e Firefly luciferase activity in HEK293T cells transiently transfected with a gamma-activated sequence-driven IFN- $\gamma$ reporter plasmid (GAS) and expression plasmids for WT or mutant SOCS1 proteins, and then stimulated with IFN- $\gamma$ for $24 \mathrm{~h}$. The results correspond to the fold-difference between the stimulated state and the unstimulated state. Results represent at least $n=4$ independent experiments. All constructs were compared with WT SOCS1. Protein expression from the transfected plasmids was confirmed by immunoblotting the cell lysates (below, one representative result). Statistics: EV $p<0.0001, \mathrm{P} 123 \mathrm{R} p<0.0001$, A9FS $^{\star} 76 p<0.0001, \mathrm{M} 161 F S^{\star} 46 p<0.0001, \mathrm{R} 22 \mathrm{~W} p=0.0011, \mathrm{Y} 154 \mathrm{H} p=0.0009$. a, b, d, e Two-tailed $p$ values were determined in an unpaired $t$ est. Data indicate mean with SD. ${ }^{\star} P \leq 0.05 ;{ }^{\star \star} P \leq 0.01 ;{ }^{\star \star \star} P \leq 0.001 ;{ }^{\star \star \star \star} P \leq 0.0001$. 


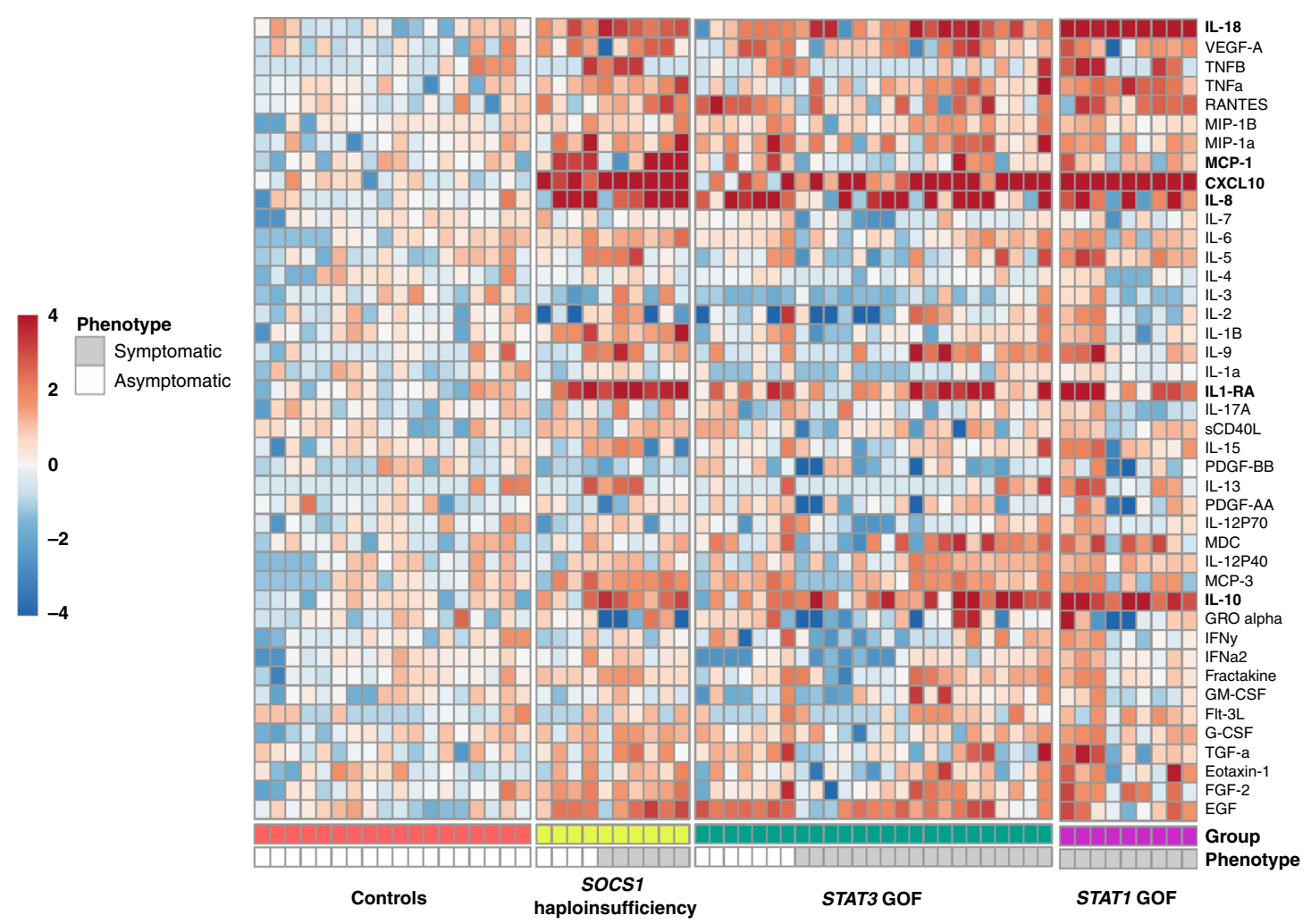

Fig. 3 Inflammatory cytokine signature in serum from patients with SOCS1 mutations. Cytokine array analysis of serum from patients with SOCS1 insufficiency, controls, patients with STAT1 GOF mutations, and patients with STAT3 GOF mutations. Gray: symptomatic patients; white: asymptomatic carriers. Decimal logarithms of the values determined in a multiplex bead assay were color-coded as follows: for each individual cytokine, median values obtained in the $17 \mathrm{HCs}$ were defined as 0 (white). The $\mathrm{X}$-fold standard deviation above this median ( 0 to +4 , coded in red) or below this median ( 0 to -4 , coded in blue) is shown with the individual squares. The color code was arbitrarily truncated at \pm 4 SDs.

nificantly higher than in control monocytes (Supplementary Fig. 7). Of note, the response to IL-21 was similar to that seen in controls in the cell line tested (A1, Supplementary Fig. 8). We next assessed the ability of mutant SOCS1 variants expressed in HEK293T cells to suppress the transcription of IFN- $\gamma$ inducible genes in a luciferasebased reporter assay by using a promoter containing an interferongamma-activated sequence (GAS). The overexpressed mutant proteins failed to suppress IFN- $\gamma$-mediated luciferase expression, whereas overexpression of the wild-type SOCS1 protein resulted in efficient inhibition (Fig. 2e). Addition of mutated proteins form of SOCS1 did not alter the inhibitory capacity of wild-type SOCS1 as observed with the four tested mutants (Supplementary Fig. 9), suggesting that the mutant proteins do not exert a dominantnegative effect. Taken together, these data indicate that SOCS1 haploinsufficiency confers hypersensitivity to, at least, IFN- $\gamma$, IL-2, and IL-4 cytokines. Consistently, an analysis of serum samples from patients revealed elevated levels of inflammatory mediators, such as IL-8, IL-10, IL-18, IL1-RA, CXCL10, and MCP-1 (Fig. 3). In contrast, the concentration of IFN- $\gamma$, IL-2, and IL-4 was not significantly increased. Interestingly, this cytokine signature was similar to that observed in individuals carrying gain-of-function (GOF) mutations in STAT1 or STAT3.

Effect of SOCS1 haploinsufficiency on T cells. Given the lymphoproliferation observed in families $\mathrm{B}$ and $\mathrm{C}$, we measured the proliferation of the patients' $\mathrm{T}$ cells in response to various stimuli in vitro. The patients' $\mathrm{T}$ cells proliferated more intensely than control $\mathrm{T}$ cells in response to stimulation with various concentrations of IL-2 but not in response to T-cell receptor stimulation with anti-CD3 antibodies (Fig. 4a). This hyperproliferation in response to IL-2 was not associated with increased expression of
CD25, the alpha chain of the IL-2 receptor and a STAT5 target gene, neither on non-activated primary $\mathrm{T}$ cells nor on activated T cells blasts (Supplementary Fig. 10), suggesting an increased receptor internalization and/or shedding of CD25 in the presence of IL-2, or that SOCS1 deficiency may be counterbalanced by another mechanism yet to be determined.

In mice, Socs1 was previously shown to be involved in regulatory $\mathrm{T}$ cell $\left(\mathrm{T}_{\text {reg }}\right)$ integrity and function, by maintaining Foxp 3 expression and by suppressing Stat 1 and Stat 3 activation ${ }^{9}$. Moreover, socs 1 deletion in Foxp $3^{+}$cells in the mouse is sufficient to induce lymph node enlargement and splenomegaly ${ }^{10}$, prompting us to analyze $\mathrm{T}_{\text {regs }}$ in patients. The mean $\mathrm{CD} 4^{+}$ $\mathrm{CD}_{25}{ }^{+} \mathrm{FOXP}^{+}{ }^{+} \mathrm{T}_{\text {regs }}$ frequency was lower in patients with SOCS1 haploinsufficiency (Fig. 4b). This reduction in $\mathrm{T}_{\text {regs }}$ frequency was not due to the expansion of memory CD4 T cells (Fig. 4c). With the exception of CTLA-4, the expression of several key $\mathrm{T}_{\text {reg }}$ markers (including FOXP3, HELIOS, and, to a lesser extent, CD25) was lower in patients' $T_{\text {regs }}$ (Fig. $4 \mathrm{~d}$ and Supplementary Fig. 11). Furthermore, the $\mathrm{T}_{\text {regs }}$ ' suppressive activity was moderately but significantly reduced in the patients' $\mathrm{T}_{\text {reg }}$ cells (A2, D1, E1) as compared with control cells (Fig. 4e and Supplementary Fig. 12). Thus, SOCS1 deficiency leads to a defective $\mathrm{T}_{\text {reg }}$ compartment. Despite SOCS1's putative role in $\mathrm{CD}^{+} \mathrm{T}$ cell differentiation ${ }^{11}$, we did not observe an obvious imbalance in Th1, Th2, Th17, and T follicular helper cells (Supplementary Fig. 13). No impact of SOCS1 deficiency on T cell apoptosis was observed (Supplementary Fig. 14).

Immunological features in healthy mutation carriers. We identified five unaffected family members carrying the same SOCS1 mutation as their affected relatives (Fig. 1a). At the time of 

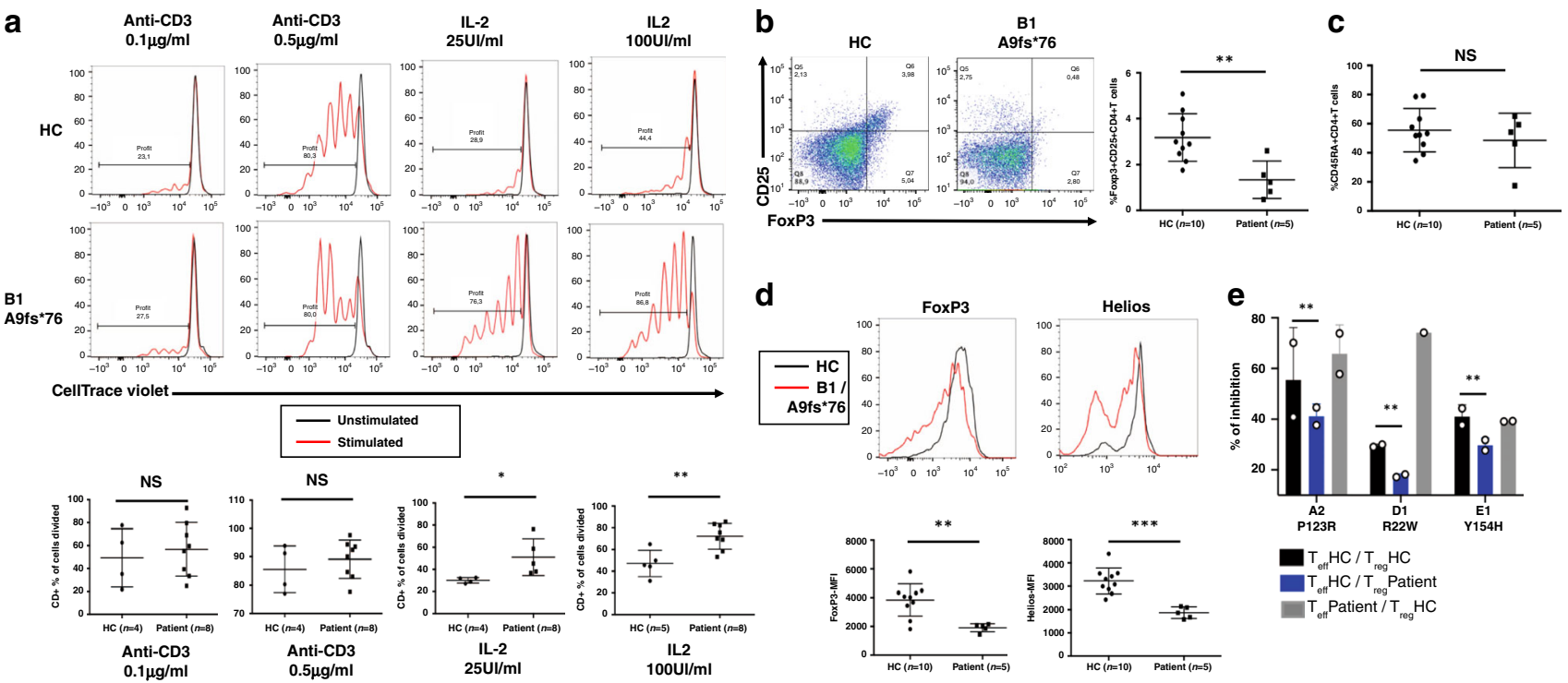

Fig. 4 Impact of SOCS1 mutations on T cell proliferation and on regulatory T cells. a Proliferation of T-cell blasts from healthy controls (HC) and patients. Day-10 T-cell blasts were stimulated or not with IL-2 $(100 \mathrm{IU} / \mathrm{ml})$, or anti-CD3-coated beads for 4 days. Proliferation was determined from the level of dilution of the CellTrace Violet dye. The panel shows proliferation of all T cells (CD3+). Top: representative histograms showing cell divisions of T-cell blasts (red peak) from an $\mathrm{HC}$ and from patient B1. Peak in black: unstimulated cells. The data are quoted as the percentage of cells having undergone at least one division. Bottom: the percentage of dividing cells from HCs and patients (data pooled from $n=4$ independent experiments including a total of four $\mathrm{HC}$, five patients with $\mathrm{IL}-225 \mathrm{UI} / \mathrm{ml}$ and eight patients with anti-CD3 or IL-2 100Ul/ml). Two-tailed $p$ values were determined in an unpaired $t$ test (IL-2 25UI/ml $p=0.0433, \mathrm{IL} 2100 \mathrm{UI} / \mathrm{ml} p=0.0038$, NS, not significant). b Left: a representative flow cytometry analysis of FoxP3 and CD25 markers in CD4+ T cells from an $\mathrm{HC}$ and from patient B1. Right: FoxP3 ${ }^{+} \mathrm{CD} 25^{+} \mathrm{CD} 4+\mathrm{T}$ cells (as a percentage of total CD4 ${ }^{+} \mathrm{T}$ cells) in the peripheral blood of $\mathrm{HCs}$ $(n=10)$ and patients $(n=5)$. c Naïve CD45RA + CD4 + T cells (as a percentage of total CD4+ T cells) in the peripheral blood of HCs $(n=10)$ and patients $(n=5)$. d Top: representative histograms of FoxP3 and Helios expression in $C D 4{ }^{+} \mathrm{CD} 25^{+} \mathrm{CD} 127$ low $\mathrm{T}$ cells from an $\mathrm{HC}$ (black) and patient $\mathrm{B} 1$ (red). Bottom: mean fluorescence intensity (MFI) of the respective $\mathrm{T}_{\text {reg }}$ cell markers in HCs $(n=10)$ and patients $(n=5)$. b-d Two-tailed $p$ values were determined in a Mann-Whitney test (Panel B $p=0.0047$; Panel D FoxP3 $p=0.008$, Helios $p=0.0007$, NS, not significant). a-d Data indicate mean with $\mathrm{SD}$, and each dot corresponds to an individual. e Suppressive activity of regulatory $T$ cells. VioBlue-labeled $T_{\text {eff }}$ cells were cultured in the absence or presence of $\mathrm{T}_{\text {reg }}$ cells from HCs and patients (A2, D1, and E1). Proliferation was determined from the level of dilution of the VioBlue dye. Graphs indicate percentages of suppression from patients. Data are from three independent experiments in duplicate with a healthy control and indicated patient tested in pairs. Data indicate mean with SD of technical replicates due to the lack of cells for more experiments. Two-tailed $p$ value was determined in a paired $t$-test between patients and controls $(p=0.0055)$. a-e ${ }^{\star} P \leq 0.05 ;{ }^{\star \star} P \leq 0.01,{ }^{\star \star \star} P \leq 0.001$.

the genetic analysis, these healthy carriers (B3, C2, D2, D3, E3) were $10,54,23,52$, and 62 years old, respectively. Four (C2, D2, D3, E3) were considered to be completely asymptomatic. One carrier (B3) developed severe asthma at 10 with an elevated serum IgE. Immunologic phenotyping performed in four carriers (B3, $\mathrm{C} 2, \mathrm{D} 2$, and E3) revealed similarities with the patients including low marginal zone and/or switch memory B-cell counts in three of them and elevated CD21 low CD38 lowB-cell count in two (Supplementary Table 1 and Supplementary Fig. 1). One (E3) of the two healthy carriers tested had autoantibodies (antinuclear and anti-SSA autoantibodies). Serum analysis from four of the carriers showed an inflammatory cytokine signature similar to patients (Fig. 3). Finally, IFN- $\gamma$-induced STAT1 phosphorylation in primary monocytes was higher than controls in two of the four tested healthy carriers (Supplementary Fig. 7). Taken together these data suggest that the immunological and functional phenotype is relatively penetrant even though the clinical phenotype is not.

In vitro and ex vivo efficacy of JAK1/JAK2 inhibition. Given that SOCS1 mutations are associated with uncontrolled JAK/ STAT activation, we tested in vitro the effect of the JAK1/2 inhibitor ruxolitinib on IFN- $\gamma$-induced STAT1 phosphorylation and on the expression of the IFN- $\gamma$-regulated gene CXCL9. Both were found reduced in the presence of ruxolitinib (Fig. 5a, b). The same effect was observed on IL-2-induced STAT5 phosphorylation in EBV-B cells (Fig. 5a) and on IL-2-induced proliferation in $\mathrm{T}$ cells as well (Fig. 5c). In contrast, ruxolitinib had no effect on anti-CD3/CD28-induced $\mathrm{T}$ cell proliferation (Fig. 5c). Finally, one patient (E1) with systemic lupus erythematosus who was clinically well controlled under mycophenolate mofetil but because of disabling digestive intolerance was switched to baricitinib (JAK1/2 inhibitor) at $2 \mathrm{mg}$ once daily followed by $2 \mathrm{mg}$ twice daily (Table 1 and Supplementary Information). Following three months of treatment, the tolerance was good while a decrease in anti-DNA autoantibodies was observed (Supplementary Fig. 15). Furthermore, IFN- $\gamma$-induced STAT1 phosphorylation in patients' primary monocytes was reduced under baricitinib as a function of the drug dosage given to the patient (Fig. 5d). These results indicate that SOCS1 haploinsufficiency is associated with the hyperactivation of JAK kinases through SOCS1-sensitive cytokine receptors-a finding thus with potential therapeutic value.

\section{Discussion}

We found that SOCS1 haploinsufficiency is associated with dominantly inherited early onset autoimmunity and lymphoproliferation in ten patients from five unrelated families. These observations highlight a key role of SOCS1 in immune homeostasis in humans. We notably demonstrated that the SOCS1 

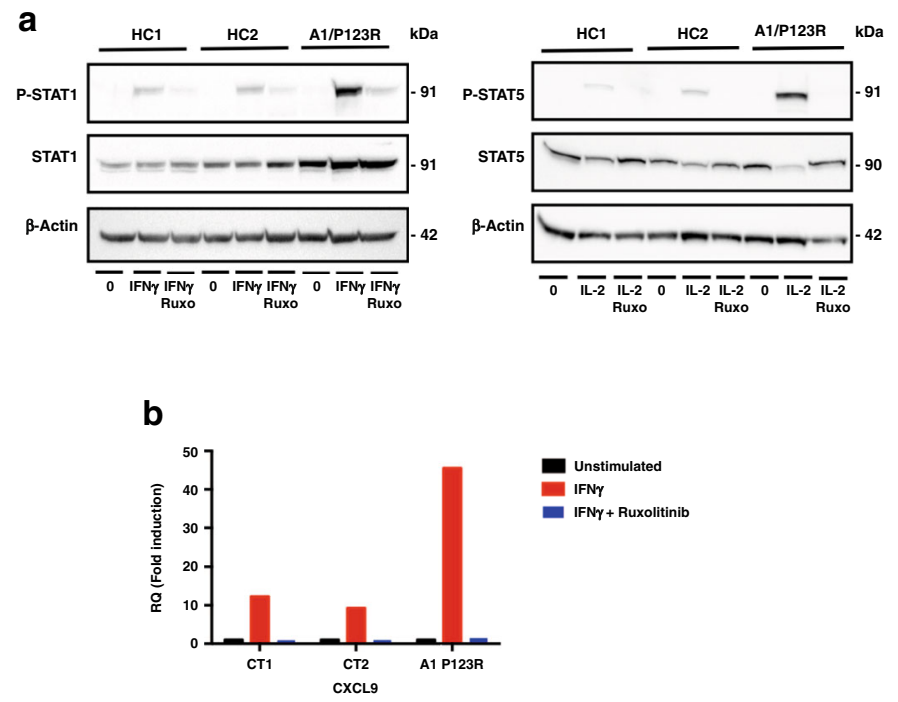

c

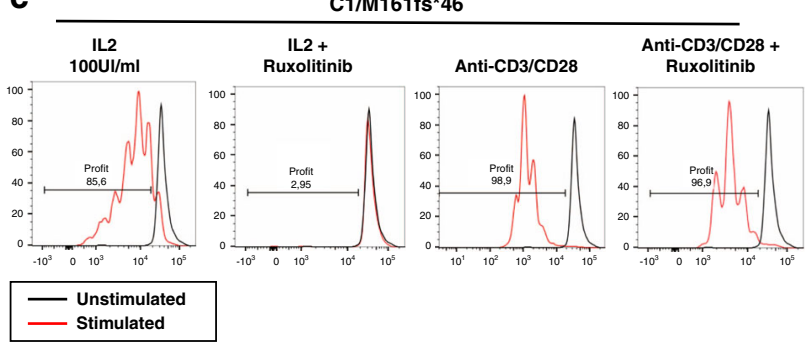

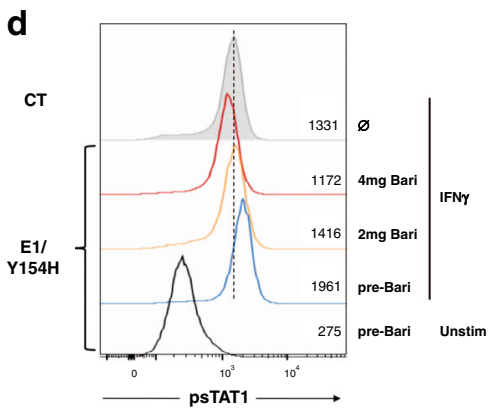

Fig. 5 In vitro and ex vivo efficacy of JAK1/JAK2 inhibition. a EBV-B cells from HCs and from patient $A 1$ were stimulated with IFN- $\gamma\left(10^{3} / \mathrm{U} / \mathrm{ml}\right.$ for $1 \mathrm{~h}$, left) and IL-2 (104 IU $/ \mathrm{ml}$ for $2 \mathrm{~h}$, right) in the presence or absence of ruxolitinib (Ruxo). Lysates were incubated with the indicated anti-P-STAT and antiSTAT antibodies, or anti-actin antibodies as a loading control. Data are representative of two independent experiments. $\mathbf{b}$ EBV-B cells from controls (CT) and from patient A1 were preincubated or not with ruxolitinib for $1 \mathrm{~h}$, and stimulated for $6 \mathrm{~h}$ with IFN- $\gamma$. mRNA expression of the STAT1-regulated gene CXCL9 was determined in a quantitative RT-PCR assay. Results represent the fold-increased expression between stimulated and unstimulated states and are normalized to endogeneous GAPDH. Experiment performed once. c Effect of in vitro treatment with ruxolitinib on T-cell proliferation. T-cell blasts from patient $\mathrm{C} 1$ were stimulated with $\mathrm{IL}-2(100 \mathrm{IU} / \mathrm{ml})$ or anti-CD3/CD28 beads, in the presence or absence of ruxolitinib. The panel shows the proliferation of all T cells $\left(\mathrm{CD}^{+}\right)$. Data are representative of two independent experiments with cells from five patients (A1, A2, B1, B2, and C1). d Primary monocytes from healthy control (CT, filled line) and $\mathrm{E} 1$ before (blue line) and under treatment by $2 \mathrm{mg}$ (orange line) or $4 \mathrm{mg}$ (red line) of baricitinib were stimulated with IFN- $\gamma$ for $15 \mathrm{~min}$ and STAT1 phosphorylation (P-STAT1) was determined by intracelular flow cytometry.

germline mutations leading to haploinsufficiency result in (i) higher JAK-STAT pathway activation in response to different cytokines, (ii) $\mathrm{T}$ cell hyperproliferation in response to IL-2, and (iii) a defective $\mathrm{T}_{\text {reg }}$ compartment. Our observation of multiple missense and frameshift mutations in patients with autoimmune features strongly suggests that these mutations are pathogenic. Furthermore, our present results in humans are in line with murine studies ${ }^{4,9,12-14}$. In particular, Socs $1^{-1-}$ mice exhibited neonatal lethality due to excessive IFN- $\gamma$ signaling and massive inflammatory infiltration ${ }^{12}$. Moreover, haploinsufficient mice survive into adulthood but develop a systemic autoimmune disease with age, reminiscent of human SLE ${ }^{15}$. Our findings are also in agreement with a recent study identifying, by whole-genome sequencing in a large primary immunodeficiencies cohort, three patients with autoimmune manifestations and SOCS1 haploinsufficiency ${ }^{16}$.

The assessment of the molecular consequences of single-gene mutations has provided a better understanding of the mechanisms underlying autoimmunity in humans ${ }^{2}$. Multiple defects can lead to a breakdown in immune tolerance, which includes the impairment of negative T-cell selection, defective lymphocyte apoptosis, a loss of $\mathrm{B}$ cell tolerance, abnormal $\mathrm{T}_{\text {reg }}$ function, uncontrolled activation of $\mathrm{T}$ cells, and accentuated immune effector cell functions ${ }^{2}$. Our observations in SOCS1 insufficiency provide mechanistic insight into the development of autoimmunity induced by cytokine hypersensitivity in immune cells following the loss of a downregulatory element. STAT1 and STAT3 GOF mutations could also be included in this category, given that they enhance cytokine signaling and function ${ }^{17-19}$. Interestingly, the clinical phenotypes of SOCS1 insufficiency and STAT3 GOF mutations have some common features (including lymphadenopathy and autoimmune cytopenia) but also possibly differences (SLE in SOCS1 insufficiency vs. enteropathy and diabetes with STAT3 GOF mutations) $)^{20,21}$. This may reflect the scope of the various cytokine signaling pathways involved ${ }^{20,21}$ We have not seen increased infection susceptibility in the SOCS1 mutant patients, but this has to be confirmed in larger cohorts.

Several mechanisms can be proposed to explain autoimmunity in the context of SOCS1 insufficiency. In mice, deletion of Socs 1 in $\mathrm{T}$ cells is both necessary and sufficient to promote autoimmunity ${ }^{13,22}$. Mechanistically, this might be due to defective $\mathrm{T}_{\text {reg }}$ development and function in the absence of SOCS1, as previously shown in the mouse ${ }^{9}$ and here in humans. However, mice with a conditional knock-out of Socs 1 in $\mathrm{T}_{\text {regs }}$ exhibit a milder autoimmune phenotype than mice in which Socs 1 has been deleted in all $\mathrm{T}$ cells-suggesting that the cytokine hypersensitivity of conventional $\mathrm{T}$ cells also contributes to autoimmunity 9,10 . A role for SOCS1 in cytokine regulation of B cells is also plausible and may account, at least in part, for the abnormal B cell phenotype. Myeloid cells are plausibly also involved, since the autoimmune phenotype is more severe when Socs 1 is knocked out in both T cells and macrophages ${ }^{23}$. Moreover, SOCS1 deficiency in dendritic cells alone leads to the production of high levels of BAFF, the aberrant expansion of B cells, the production of autoreactive antibody, and systemic autoimmunity ${ }^{13}$. Hence, given the widespread expression of SOCS1 and the cytokines' orchestrating role in the immune system, several cell types probably contribute to autoimmunity in the context of SOCS1 haploinsufficiency.

Of the 15 individuals with a heterozygous SOCS1 mutation studied here, five (including four adults) were healthy carriers $(33 \%)$. This incomplete clinical penetrance is frequently observed 
in autosomal-dominant primary immunodeficiencies caused by haploinsufficiency (e.g. CTLA-4 $4^{24,25}$, IKZF1/IKAROS ${ }^{26}$ or in TNFRSF6/FAS deficiencies ${ }^{27}$, which have penetrance of $67 \%$, $65 \%$, and $30 \%$, respectively). The age at onset differs in individuals carrying the same mutation, and the clinical manifestations sometimes appear as late as the sixth decade of life ${ }^{25,26}$. This incomplete penetrance may indicate the requirement for additional genetic ${ }^{28}$ or epigenetic events and/or the involvement of infectious/environmental events to induce full clinical expression of the disease $\mathrm{e}^{29}$.

Several monogenic primary immunodeficiencies have been linked to an increased risk of malignancies ${ }^{30}$. This could be related to treatments with immunosuppressants and/or the gene product's role in tumor surveillance ${ }^{27}$. Somatic loss-of-function mutations in SOCS1 (including 2 mutated residues found in families A and E: P123 and Y154,8) are frequently observed in Bcell or Hodgkin lymphoma, and are associated with activation of the JAK-STAT pathway in tumor cells ${ }^{31-33}$. It is noteworthy that one patient in family $\mathrm{B}$ developed Hodgkin lymphoma at the age of 34, whereas benign lymphoproliferation was seen in two other patients. This observation should prompt the physicians to carefully monitor patients with SOCS1 deficiency and their healthy carrier relatives.

SOCS1-insufficient patients have been treated with various nonspecific immunosuppressive agents, some of which are associated with toxic effects. Given the molecular etiology of the disease, treatment with drugs inhibiting the JAK signaling pathway might constitute a more specific treatment option ${ }^{34}$. We found indeed that in vitro, the JAK1/2 inhibitor ruxolitinib strongly inhibited $\mathrm{T}$ cell hyperproliferation and cytokinemediated hyperphosphorylation of STAT1 and STAT5, without affecting the antigen-receptor-mediated response. These results were confirmed ex vivo in a patient with systemic lupus erythematosus treated by the JAK1/2 inhibitor baricitinib that was associated with an in vivo decrease of anti-DNA antibodies. Therefore, JAK inhibitors may be targeted therapies of value for SOCS1-insufficient patients. Moreover, SOCS1 itself is an attractive therapeutic target; SOCS1 agonists are being tested for efficacy in diseases mediated by IFN- $\gamma$, IL-2, or IL-4, including autoimmune and allergic disorders ${ }^{35,36}$.

In conclusion, our results show that SOCS1 haploinsufficiency is a recognized cause of early onset autoimmunity. Our results also provide mechanistic insights into the development of autoimmunity induced by cytokine hypersensitivity in immune cellsopening up avenues for personalized treatments in the field of autoimmunity.

\section{Methods}

Study participants. All study participants and their parents provided written informed consent. The study was performed in accordance with the 1975 Declaration of Helsinki and subsequent revisions, and was approved by the local institutional review board (CPP Ile de France II (Paris, France), the French Advisory Committee on Data Processing in Medical Research (Paris, France) and the Ethics committee, University of Freiburg, Germany (No. 409/16). The probands (patients A1, A2, B1, B2, C1, D1, and E1) were included in clinical studies of early onset autoimmune diseases by three independent institutions (Imagine Institute (Paris, France), Lyon Hospital (Lyon, France), and Freiburg Hospital (Freiburg, Germany)).

Genetic analysis. Samples of DNA were prepared from the patients' whole peripheral blood, using standard extraction methods. After quality control, genomic DNA $(3 \mu \mathrm{g})$ intended for WES was captured using an in-solution enrichment method (Human All Exon v5-50 Mb, Agilent Technologies, CA, USA). Exomeenriched libraries ( 20,000 targeted genes) were prepared on an automated system, using the NGSx robot (Perkin Elmer Inc, MA, USA) and the Bravo robot (Agilent Technologies, CA, USA) respectively, according to the manufacturers' instructions (SureSelect, Agilent Technologies). After normalization and quality control, exome-enriched libraries were sequenced on a HiSEQ 2000 system (Illumina Inc., CA, USA) as paired-end $100 \mathrm{~b}$ reads. The mean sequencing coverage was at least 60 to 70x for each sample. Image analysis and base calling were performed using the Illumina Real Time Analysis Pipeline. Sequence quality parameters were assessed daily throughout the 12-day sequencing run. Sequences were aligned with the hg19 reference human genome, using the Burrows-Wheeler Aligner. Downstream processing was carried out with the Genome Analysis Toolkit (GATK), SAMtools, and Picard, in line with documented best practices (http://www.broadinstitute.org/gatk/ guide/topic?name=best-practices). Variants were called with the GATK Unified Genotyper. All calls with a read coverage of $\leq 2 \mathrm{X}$ or a Phredscaled SNP quality score of $\leq 20$ were removed from the analysis. All variants were annotated using a software system developed by the Paris Descartes University Bioinformatics platform. All the annotation procedures were based on the latest release of the Ensembl database.

Whole-genome sequencing was performed by Edinburgh Genomics. Genomic DNA was prepared from whole blood samples from probands and parents, using the Qiagen Mini Kit according to the manufacturer's instructions. Genomic DNA (gDNA) samples were evaluated for quantity using Quant-IT Picogreen reagent, Lambda Standard DNA, and a Spectramax XPS Gemini plate reader. Quality was evaluated using AATI Fragment analyzer and the Standard Sensitivity Genomic DNA Analysis kit. Samples with $>1000 \mathrm{ng}$ and a quality score of $>5$ passed the sample quality control. Sequencing libraries were prepared using a specific Illumina SeqLab TruSeq Nano High Throughput kit. A total of $200 \mathrm{ng}$ of gDNA was normalized and sheared to a $450 \mathrm{bp}$ mean insert size. TruSeq adapters were ligated onto the ends of each fragment prior to PCR amplification. Caliper GX Touch with an HT DNA HI SENSE Reagent Kit was used to produce mean fragment sizes of $530-950 \mathrm{bp}$. Libraries were normalized to $1.5 \mathrm{nM}$ and sequenced at $300 \mathrm{pM}$. Demultiplexing was performed using bcl2fastq.

The raw sequencing data were quality-checked using FastQC and MultiQC, and subsequently aligned with the GRCh 37 hs $37 \mathrm{~d} 5$ reference using the Edico Dragen V2 single-sample workflow. Indels and SNPs were called using the same workflow on an FPGA AWS instance. The resulting variant call format files were then uploaded to the Congenica platform via the latter's Upload Client (v1.7.4) for annotation and causal variant prioritization. Variants were annotated using the Ensembl Variant Effect Predictor and scored for disease causality using Exomiser For each variant in the proband, we determined the mode of inheritance in the family trio (de novo, autosomal recessive homozygous, autosomal recessive compound heterozygous, or X-linked recessive). DoNovoGear was used for the joint analysis of samples within a trio, in order to detect de novo mutations. Variants with qualifying genotypes under each mode of inheritance were ranked using the Exomiser combined score and evaluated for rarity (MAF in ExAC and UK10K), pathogenicity (Polyphen2 and SIFT scores), genic intolerance to functional variation (RVIS and pLI scores) and biological relevance in the context of the proband's phenotype (using a Phenotype Consensus Analysis and a literature search). A rule-based scheme was used to identify candidate genes.

The pathogenicity of the identified variants was defined according to the minor allele frequency in the general population according to the GnomAD database (https://gnomad.broadinstitute.org/); and the Combined Annotation-Dependent Depletion score ${ }^{37}$.

Sanger sequencing was performed to confirm the next-generation sequencing results and to analyze each mutation's familial segregation. The primers used for PCR are reported in Supplementary Table 1. Purified PCR products were directly sequenced using BigDye Terminators (version 1.1) and a 3500xL Genetic Analyzer (Applied Biosystems).

Three-dimensional structure modeling. The model of the human SOCS1 3D structure was built on the basis of the experimental 3D structure of chicken SOCS1 (71\% sequence identity, pdb 6C7Y), according to the alignment given in Supplementary Fig. 2. The possible path of a phosphotyrosine peptide, represented in purple in Fig. 1d (bottom view), with the side chain of the phosphotyrosine shown, was taken from the experimental 3D structure of the SOCS3:gp130 complex (Protein Data Bank 2HMH). The model of the whole JAK1/SOCS1/elongin B/ elongin C complex shown in Fig. 1d (top) is based on the chicken SOCS1/JAK1 (Protein Data Bank 6C7Y) and X. laevis SOCS1/Elongin B/Elongin C (Protein Data Bank 6C5X) 3D structures. Modeling was made using Modeller v9.22 ${ }^{38}$ and 3D vizualisation using Chimera ${ }^{39}$.

Cell culture. Peripheral blood mononuclear cells (PBMCs) were isolated using Ficoll separation (Lonza) and then cultured in Panserin 401 medium (Dutcher) with $5 \%$ human male $\mathrm{AB}$ serum (BioWest), $2 \mathrm{mM}$ L-glutamine, $100 \mathrm{U} / \mathrm{ml}$ penicillin, and $100 \mu \mathrm{g} / \mathrm{ml}$ streptomycin (Thermo Fisher Scientific) at $37^{\circ} \mathrm{C}$ in a humidified $5 \% \mathrm{CO}_{2}$ incubator. Expansion of $\mathrm{T}$ cell blasts was obtained by incubating PBMCs for $72 \mathrm{~h}$ with CD3/CD28 beads (LifeTechnologies) in complete Panserin 401. After 3 days, dead cells were removed by Ficoll-Plaque density gradient, and blasts were expanded in complete Panserin supplemented with IL-2 (100 IU/ml). Epstein-Barr-virus (EBV)-transformed B lymphoblastoid cell lines (EBV-B cells) were generated from PBMCs obtained from patients (A1, B1, and D1) and HCs using standard methods and then cultured in RPMI 1640 (LifeTechnologies) supplemented with $10 \%$ fetal bovine serum (FBS) and penicillin/ streptomycin. Human embryonic kidney (HEK) 293T cells were cultured in DMEM (LifeTechnologies) supplemented with 10\% FBS and penicillin/ streptomycin. 
Flow cytometry analysis. For surface staining, PBMCs (either freshly isolated or thawed after storage in liquid nitrogen) were washed with cold PBS and then incubated for $30 \mathrm{~min}$ at $4^{\circ} \mathrm{C}$ (in the dark) with appropriate fluorochrome-labeled monoclonal antibodies. For intracellular staining, PBMCs were fixed and permeabilized after surface staining using the FoxP3 staining kit (eBioscience) according to the manufacturer's instructions, and then incubated with anti-FOXP3 and anti-CTLA4 for $30 \mathrm{~min}$ on ice. The antibodies used for flow cytometry are listed in Supplementary Table 2. All samples were measured using flow cytometry (Becton Dickinson LSRFortessa X-20) and the data were analyzed using the FlowJo software (TreeStar).

Expression plasmids, mutagenesis, and transfection experiments. Complementary DNA from WT human SOCS1 (SOCS1; NM_003745) was subcloned into the mammalian expression vector pCMV-HA-tagged (Clontech). The indicated SOCS1 mutants were generated using the Q5 site-directed mutagenesis kit protocol (NEB). All cDNA sequences were confirmed by Sanger sequencing. Wildtype or mutant SOCS1 plasmids were transfected into HEK293T cells by transient transfection using lipofectamine 2000 (Life Technologies).

Western blot analysis. The EBV-B cell lines were exposed to various stimuli prior to protein extraction. The following optimal stimulation conditions were used: 60 min with $10^{3} \mathrm{IU} / \mathrm{ml} \mathrm{IFN}-\gamma, 120 \mathrm{~min}$ with $10^{4} \mathrm{IU} / \mathrm{ml} \mathrm{IL}-2,60 \mathrm{~min}$ with $10 \mathrm{ng} / \mathrm{ml} \mathrm{IL-}$ 4, $45 \mathrm{~min}$ with 10 and $100 \mathrm{ng} / \mathrm{ml} \mathrm{IL-21,} \mathrm{and} 60 \mathrm{~min}$ with $100 \mathrm{ng} / \mathrm{ml} \mathrm{IL-6.} \mathrm{For}$ blocking experiments, cells were preincubated with ruxolitinib $(500 \mathrm{nM})$ for an hour prior to stimulation. Cell activation was blocked with cold 1X PBS, and then lysed in RIPA lysis buffer supplemented with a protease and phosphatase inhibitor cocktail (ThermoFisher Scientific). The extracted proteins were quantified using a Pierce BCA assay kit (ThermoFisher Scientific), subjected to SDS-PAGE, and transferred to a PVDF membrane. For the extraction of nuclear and cytoplasmic proteins, EBV-B cells were stimulated with INF $\gamma$ for $1 \mathrm{~h}$ or IL-2 for $2 \mathrm{~h}$ and extracted with NE-PER Nuclear and Cytoplasmic Extraction Regents (ThermoFisher Scientific), according to the manufacturer's instructions. After blocking, the membrane was incubated overnight with the following primary antibodies: antiSOCS1 (Cell Signaling Technology), anti-phosphorylated STAT1 Y701 (P-STAT1, Cell Signaling Technology), anti-STAT1 (Cell Signaling Technology), anti-PSTAT3Y705 (Cell Signaling Technology), anti-STAT3 (Cell Signaling Technology), anti-P-STAT5 ${ }^{\mathrm{Y} 694}$ (Cell Signaling Technology), anti-STAT5 (Cell Signaling Technology), anti-P-STAT6 ${ }^{\text {Y641 }}$ (Cell Signaling Technology), anti-STAT6 (Cell Signaling Technology), anti- $\alpha$-tubulin (Cell Signaling Technology), anti- $\beta$-actin (ThermoFisher Scientific), anti-GAPDH (Cell Signaling Technology), anti-HA (Cell Signaling Technology), and anti-lamin A/C (Cell Signaling Technology). The results were visualized by chemiluminescence using species-specific HRP-linked secondary antibodies.

Real-time quantitative RT-PCR. The EBV-B cells were stimulated for $6 \mathrm{~h}$ with $10^{3}$ $\mathrm{IU} / \mathrm{ml}$ IFN- $\gamma$ or $10^{4} \mathrm{IU} / \mathrm{ml}$ IL-2. Total RNA was isolated using the RNeasy Mini Kit (Qiagen), and cDNA was prepared using the Quantitect Reverse Transcription Kit (Qiagen) after depletion of genomic DNA. Real-time quantitative PCR was performed using the LightCycler VIIA7 System (Roche). Gene transcript levels were normalized against an endogenous GAPDH control. The results were quoted as the fold-increase in expression, relative to the unstimulated state. Each experiment was performed in triplicate in at least two independent experiments.

T-helper cell cytokine assays. Peripheral blood mononuclear cells from patients and HCs were stimulated ex vivo with phorbol myristate acetate $(50 \mathrm{ng} / \mathrm{ml}$; SigmaAldrich) and ionomycin $(1 \mu \mathrm{g} / \mathrm{ml}$; Sigma-Aldrich) for $4 \mathrm{~h}$ in the presence of brefeldin A (GolgiPlug, 1:1000; BD Biosciences), surface-stained, fixed/permeabilized (with a Cytofix/Cytoperm plus Kit, BD Biosciences) and stained with anti-IL-4, IL17A and IFN- $\gamma$ antibodies (Supplementary Table 2). The fraction of Th2, Th17, and Th1 cells was respectively determined (using flow cytometry) as the percentages of IL- $4^{+}, \mathrm{IL}_{17 \mathrm{~A}}+$, and IFN- $\gamma^{+}$cells among $\mathrm{CD} 4^{+} \mathrm{CD} 45 \mathrm{RO}^{+}$cells.

STAT 1 phosphorylation in monocytes. PBMCs of patients and healthy controls were left unstimulated or stimulated with IFN- $\gamma(1000 \mathrm{IU} / \mathrm{ml})$ for $15 \mathrm{~min}$ and subsequently fixed, permeabilized (Phosflow Lyse/Fix buffer, BD and Phosflow Perm buffer III, BD) and stained with anti-CD14 or anti-CD33 and anti-PSTAT1 ${ }^{\text {Y701 }}$ antibodies (Supplementary Table 2). The extent of P-STAT1 upon stimulation was determined by flow cytometry as the mean fluorescence intensity (MFI) in $\mathrm{CD}_{1} 4^{+}$cells or $\mathrm{CD}_{3} 3^{+}$cells.

Luciferase reporter assays. HEK293T cells were dispensed into a 96-well cellculture plate and transiently cotransfected using Lipofectamine 2000 (Life Technologies) with a luciferase reporter vector under the control of the interferongamma-activated (GAS) promoter (Promega), a Renilla control vector (Promega), and plasmids expressing either WT SOCS1, the various mutant SOCS1 CDNAs, or a mock vector. $6 \mathrm{~h}$ after transfection, the cells were transferred back into medium containing 10\% FBS and cultured for $24 \mathrm{~h}$. Transfected cells were then stimulated (or not) with IFN- $\gamma\left(10^{3} \mathrm{IU} / \mathrm{ml}\right)$ for $24 \mathrm{~h}$ and subjected to luciferase assays with the
Dual-Glo luciferase assay system (Promega). Experiments were performed in triplicate, and firefly luciferase activity was normalized against the level of Renilla luciferase activity. The data are expressed as fold-induction, relative to unstimulated cells.

Proliferation assay. Expansion of T cell blasts was obtained by incubating PBMCs for $72 \mathrm{~h}$ with anti CD3/CD28 beads in complete Panserin 401 medium. After 3 days, dead cells were removed by Ficoll-Plaque density gradient, and T-cell blasts were expanded in complete Panserin supplemented with IL-2 (100 IU/ml). Day 10 T-cell blasts were starved of IL-2 for $72 \mathrm{~h}$, washed, incubated with CellTrace violet reagent (Invitrogen) for $8 \mathrm{~min}$ at $37^{\circ} \mathrm{C}$ in the dark, and washed twice more. A total of $2 \times 10^{5}$ cells were seeded into 96 -well plates and subjected to different stimuli (plate-bound anti-CD3 antibody (Invitrogen), soluble anti-CD3/CD28 beads (eBioscience), IL-2 at the concentration indicated in the figures) in the presence of absence of ruxolitinib $(500 \mathrm{nM})$. The cells were cultured for 4 days, washed with PBS, and stained with anti-CD3, CD4, CD8, CD25, and CD69 antibodies prior to flow cytometry measurement.

$\mathbf{T}_{\text {reg }}$ cell suppression assay. PBMCs were incubated for $30 \mathrm{~min}$ at $4{ }^{\circ} \mathrm{C}$ with specific, labeled monoclonal antibodies, washed, and then sorted using an ARIA II cytometer (BD Biosciences). Naïve T effector cells (Teff) were defined as $\mathrm{CD} 3^{+}$ $\mathrm{CD} 4{ }^{+} \mathrm{CD} 25^{-} \mathrm{CD} 127^{+} \mathrm{CD} 45 \mathrm{RA}^{+} \mathrm{T}$ cells, Tregs were defined as $\mathrm{CD} 3^{+} \mathrm{CD} 4^{+}$ $\mathrm{CD} 25^{+} \mathrm{CD} 127^{-} \mathrm{T}$ cells, and dendritic cells (DCs) were defined as CD3-CD4 CD14-CD16-CD11c $\mathrm{c}^{+}$cells. After cell sorting, Teff was washed and stained with CellTrace CFSE. Next, the cells were washed, incubated with DCs (Teff:DC ratio 1:0.4), Tregs (Teff:Treg ratio 1:0.5) and stimulated by Staphylococcus enterotoxin E $(0.2 \mathrm{ng} / \mathrm{ml})$. The cells were cultured for 5 days in complete Panserin medium (Dutscher) in 96-well plates and the proportion of proliferating cells was measured with a MACSquant system (Miltenyi). The data were analyzed with FlowJo software. The percentage of suppression was calculated with the following formula: [ $\log 2(y)$ of (Teff cells alone) $-\log 2(y)$ of (Teff + Treg cells])/ $\log 2(y)$ of (Teff cells alone) $\times 100$. The $y$ value corresponds to the mean fluorescence intensity of the CFSE of the whole Teff cell population, divided by the mean fluorescence intensity of the CFSE of undivided Teff cells ${ }^{40}$.

Cytokine assays. Serum levels of the cytokines EGF, eotaxin-1, FGF-2, Flt-3L, fractalkine, G-CSF, GM-CSF, GRO(alpha), IFN-alpha2, IFN- $\gamma$, IL-1 $\alpha$, IL-1 $\beta$, IL1ra, IL-2, IL-3, IL-4, IL-5, IL-6, IL-7, IL-8, IL-9, IL-10, IL-12 (p40), IL-12 (p70), IL13, IL-15, IL-17A, IL-18, IP-10, MCP-1, MCP-3, MDC, MIP-1 $\alpha$, MIP-1 $\beta$, PDGFAA, PDGF-AB/BB, RANTES, sCD40L, TGF $\alpha$, TNF $\alpha$, TNF $\beta$, VEGF-A were determined using a multiplex bead assay (Human Cytokine Array/Chemokine Array 42-Plex with IL-18 (HD42), Eve Technologies; Canada).

Statistics. All statistical analyses were performed using GraphPad Prism (version 6). As indicated in the Figures, results were analyzed in a two-tailed, unpaired or paired Student's $t$ test, or a two-sided Mann-Whitney $\mathrm{U}$ test. For all analyses, the threshold for statistical significance was set to $p<0.05$.

Reporting summary. Further information on research design is available in the Nature Research Reporting Summary linked to this article.

\section{Data availability}

SOCS1 variants sequences are deposited in the ClinVar database [https://www.ncbi.nlm. nih.gov/clinvar?term=603597[MIM]] with the following accession codes SCV001430913, SCV001430914, SCV001430915, SCV001430916, SCV001430917. Gnomad database (https://gnomad.broadinstitute.org/) was used to calculate the minor allele frequency of all the mutations. PopViz webserver (http://shiva.rockefeller.edu/PopViz/) was used to calculate the CADD score and the mutation significance (MSC) cutoff of all the mutations. The datasets generated and/or analyzed during the current study are available from the corresponding authors on reasonable request. Source data are provided with this paper.

Received: 1 March 2020; Accepted: 8 September 2020; Published online: 21 October 2020

\section{References}

1. Goodnow, C. C. Multistep pathogenesis of autoimmune disease. Cell 130, 25-35 (2007).

2. Grimbacher, B., Warnatz, K., Yong, P. F. K., Korganow, A.-S. \& Peter, H.-H. The crossroads of autoimmunity and immunodeficiency: Lessons from polygenic traits and monogenic defects. J. Allergy Clin. Immunol. 137, 3-17 (2016).

3. Villarino, A. V., Kanno, Y. \& O'Shea, J. J. Mechanisms and consequences of Jak-STAT signaling in the immune system. Nat. Immunol. 18, 374-384 (2017). 
4. Yoshimura, A., Naka, T. \& Kubo, M. SOCS proteins, cytokine signalling and immune regulation. Nat. Rev. Immunol. 7, 454-465 (2007).

5. Liau, N. P. D. et al. The molecular basis of JAK/STAT inhibition by SOCS1. Nat. Commun. 9, 1558 (2018).

6. Itan, Y. et al. The mutation significance cutoff: gene-level thresholds for variant predictions. Nat. Methods 13, 109-110 (2016).

7. Juskevicius, D. et al. Mutations of CREBBP and SOCS1 are independent prognostic factors in diffuse large B cell lymphoma: mutational analysis of the SAKK 38/07 prospective clinical trial cohort. J. Hematol. Oncol. 10, 70 (2017).

8. Mareschal, S. et al. Whole exome sequencing of relapsed/refractory patients expands the repertoire of somatic mutations in diffuse large B-cell lymphoma. Genes Chromosomes Cancer 55, 251-267 (2016).

9. Takahashi, R. et al. SOCS1 is essential for regulatory $\mathrm{T}$ cell functions by preventing loss of Foxp3 expression as well as IFN-\{gamma\} and IL-17A production. J. Exp. Med. 208, 2055-2067 (2011).

10. Takahashi, R., Nakatsukasa, H., Shiozawa, S. \& Yoshimura, A. SOCS1 Is a key molecule that prevents regulatory $\mathrm{T}$ cell plasticity under inflammatory conditions. J. Immunol. 199, 149-158 (2017).

11. Fujimoto, M. et al. A regulatory role for suppressor of cytokine signaling-1 in $\mathrm{T}(\mathrm{h})$ polarization in vivo. Int. Immunol. 14, 1343-1350 (2002).

12. Alexander, W. S. et al. SOCS1 is a critical inhibitor of interferon gamma signaling and prevents the potentially fatal neonatal actions of this cytokine. Cell 98, 597-608 (1999).

13. Hanada, T. et al. Suppressor of cytokine signaling-1 is essential for suppressing dendritic cell activation and systemic autoimmunity. Immunity 19, 437-450 (2003).

14. Wang, H., Wang, J. \& Xia, Y. Defective suppressor of cytokine signaling 1 signaling contributes to the pathogenesis of systemic lupus erythematosus. Front Immunol. 8, 1292 (2017).

15. Fujimoto, M. et al. Inadequate induction of suppressor of cytokine signaling-1 causes systemic autoimmune diseases. Int. Immunol. 16, 303-314 (2004).

16. Thaventhiran, J. E. D. et al. Whole-genome sequencing of a sporadic primary immunodeficiency cohort. Nature 1-6. https://doi.org/10.1038/s41586-0202265-1 (2020).

17. Flanagan, S. E. et al. Activating germline mutations in STAT3 cause earlyonset multi-organ autoimmune disease. Nat. Genet. 46, 812-814 (2014).

18. Liu, L. et al. Gain-of-function human STAT1 mutations impair IL-17 immunity and underlie chronic mucocutaneous candidiasis. J. Exp. Med. 208, 1635-1648 (2011)

19. van de Veerdonk, F. L. et al. STAT1 mutations in autosomal dominant chronic mucocutaneous candidiasis. N. Engl. J. Med. 365, 54-61 (2011)

20. Milner, J. D. et al. Early-onset lymphoproliferation and autoimmunity caused by germline STAT3 gain-of-function mutations. Blood 125, 591-599 (2015).

21. Haapaniemi, E. M. et al. Autoimmunity, hypogammaglobulinemia, lymphoproliferation, and mycobacterial disease in patients with activating mutations in STAT3. Blood 125, 639-648 (2015).

22. Marine, J. C. et al. SOCS1 deficiency causes a lymphocyte-dependent perinatal lethality. Cell 98, 609-616 (1999).

23. Chong, M. M. W., Metcalf, D., Jamieson, E., Alexander, W. S. \& Kay, T. W. H. Suppressor of cytokine signaling-1 in T cells and macrophages is critical for preventing lethal inflammation. Blood 106, 1668-1675 (2005).

24. Kuehn, H. S. et al. Immune dysregulation in human subjects with heterozygous germline mutations in CTLA4. Science 345, 1623-1627 (2014).

25. Schwab, C. et al. Phenotype, penetrance, and treatment of 133 cytotoxic Tlymphocyte antigen 4-insufficient subjects. J. Allergy Clin. Immunol. https:// doi.org/10.1016/j.jaci.2018.02.055 (2018).

26. Kuehn, H. S. et al. Loss of B cells in patients with heterozygous mutations in IKAROS. N. Engl. J. Med. 374, 1032-1043 (2016).

27. Neven, B. et al. A survey of 90 patients with autoimmune lymphoproliferative syndrome related to TNFRSF6 mutation. Blood 118, 4798-4807 (2011).

28. Magerus-Chatinet, A. et al. Onset of autoimmune lymphoproliferative syndrome (ALPS) in humans as a consequence of genetic defect accumulation. J. Clin. Invest. 121, 106-112 (2011).

29. Rieux-Laucat, F. et al. Lymphoproliferative syndrome with autoimmunity: a possible genetic basis for dominant expression of the clinical manifestations. Blood 94, 2575-2582 (1999).

30. Mayor, P. C. et al. Cancer in primary immunodeficiency diseases: cancer incidence in the United States immune deficiency network registry. J. Allergy Clin. Immunol. 141, 1028-1035 (2018).

31. Mottok, A., Renné, C., Willenbrock, K., Hansmann, M.-L. \& Bräuninger, A. Somatic hypermutation of SOCS1 in lymphocyte-predominant Hodgkin lymphoma is accompanied by high JAK2 expression and activation of STAT6. Blood 110, 3387-3390 (2007).
32. Mottok, A. et al. Inactivating SOCS1 mutations are caused by aberrant somatic hypermutation and restricted to a subset of B-cell lymphoma entities. Blood 114, 4503-4506 (2009).

33. Melzner, I. et al. Biallelic mutation of SOCS-1 impairs JAK2 degradation and sustains phospho-JAK2 action in the MedB-1 mediastinal lymphoma line. Blood 105, 2535-2542 (2005).

34. Forbes, L. R. et al. Jakinibs for the treatment of immune dysregulation in patients with gain-of-function signal transducer and activator of transcription 1 (STAT1) or STAT3 mutations. J. Allergy Clin. Immunol. 142, 1665-1669 (2018).

35. Durham, G. A., Williams, J. J. L., Nasim, M. T. \& Palmer, T. M. Targeting SOCS proteins to control JAK-STAT signalling in disease. Trends Pharmacol. Sci. 40, 298-308 (2019).

36. Sharma, J. \& Larkin, J. Therapeutic implication of SOCS1 modulation in the treatment of autoimmunity and cancer. Front Pharm. 10, 324 (2019).

37. Zhang, P. et al. PopViz: a webserver for visualizing minor allele frequencies and damage prediction scores of human genetic variations. Bioinformatics 34, 4307-4309 (2018)

38. Webb, B. \& Sali, A. Comparative protein structure modeling using MODELLER. Curr. Protoc. Bioinform. 54, 5.6.1-5.6.37 (2016).

39. Pettersen, E. F. et al. UCSF Chimera-a visualization system for exploratory research and analysis. J. Comput. Chem. 25, 1605-1612 (2004).

40. Zaragoza, B. et al. Suppressive activity of human regulatory $\mathrm{T}$ cells is maintained in the presence of TNF. Nat. Med. 22, 16-17 (2016).

\section{Acknowledgements}

The authors are grateful for the contribution of Anne Rensing-Ehl, Maria Elena Maccari, Sarah Grün, Myriam Lorenz and Lawrence J Petherbridge. The study was supported by the Institut National de la Santé et de la Recherche Médicale (INSERM) and by government grants managed by the Agence National de la Recherche as part of the "Investment for the Future" program (ANR-10-IAHU-01 and ANR-18-RHUS-0010), the Ligue Contre le Cancer-Comité de Paris, Fondation ARC pour la recherche sur le CANCER, the Centre de Référence Déficits Immunitaires Héréditaires (CEREDIH), the Agence National de la Recherche (ANR-14-CE14-0026-01 "Lumugene" to F.R.L.; ANR18-CE17-0001 “Action”to BN), the German Reseach Foundation (DFG) under German's Excellence strategy (CIBBS-EXC-2189-Project ID 390939984), the German Ministry for Education and Research (BMBF grant GAIN FSE2018-041) and a reseach grant from UCB Pharma (to SE). J.H. was a recipient of an INSERM "poste d'acceuil" program, and from an Institut Imagine $\mathrm{MD}-\mathrm{PhD}$ fellowship program supported by the Fondation Bettencourt Schueller. M.H. was supported by the Berta-Ottenstein clinician scientist program of the Faculty of Medicine, University of Freiburg.

\section{Author contributions}

J.H., C.C., S.E., F.R.L., and A.B. conceived and designed the study and had full access to all of the data in the study and take responsibility for the integrity of the data and the accuracy of the data analysis. J.H., C.C., M.T., M.-C.S., M.V., S.V., M.M, A.-L.M, K.S., and F.M. performed experimental studies. I.R. was in charge of the biobanking. I.C. performed three-dimensional analysis. A.S., I.C., M.P., M.A., K.S.E., J.K., and H.A. analyzed sequencing raw data and discussed results. J.-C.L., J.-F.V., A.N., N.A., I.D., M.F., B.M., Ca.P., N.F., E.R., T.R.L, G.E.-D., provided clinical cases and performed clinical routine investigations. Ce.P. provided the images of biopsy. J.H. and C.C. drafted the paper. J.H., C.C., M.T., S.E., B.N., A.F., F.R.L., T.W., G.L., D.S., M.H., and A.B. did the data analysis, and all authors critically revised the manuscript for important intellectual content and gave final approval for the version to be published. All authors agree to be accountable for all aspects of the work in ensuring that questions related to the accuracy or integrity of any part of the work are appropriately investigated and resolved.

\section{Competing interests}

The authors declare no competing interests.

\section{Additional information}

Supplementary information is available for this paper at https://doi.org/10.1038/s41467 020-18925-4.

Correspondence and requests for materials should be addressed to A.B. or F.R.-L.

Peer review information Nature Communications thanks Sandra Nicholson and the other, anonymous reviewer(s) for their contribution to the peer review of this work.

Reprints and permission information is available at http://www.nature.com/reprints

Publisher's note Springer Nature remains neutral with regard to jurisdictional claims in published maps and institutional affiliations. 
Open Access This article is licensed under a Creative Commons Attribution 4.0 International License, which permits use, sharing, adaptation, distribution and reproduction in any medium or format, as long as you give appropriate credit to the original author(s) and the source, provide a link to the Creative Commons license, and indicate if changes were made. The images or other third party material in this article are included in the article's Creative Commons license, unless indicated otherwise in a credit line to the material. If material is not included in the article's Creative Commons license and your intended use is not permitted by statutory regulation or exceeds the permitted use, you will need to obtain permission directly from the copyright holder. To view a copy of this license, visit http://creativecommons.org/ licenses/by/4.0/.

(C) The Author(s) 2020

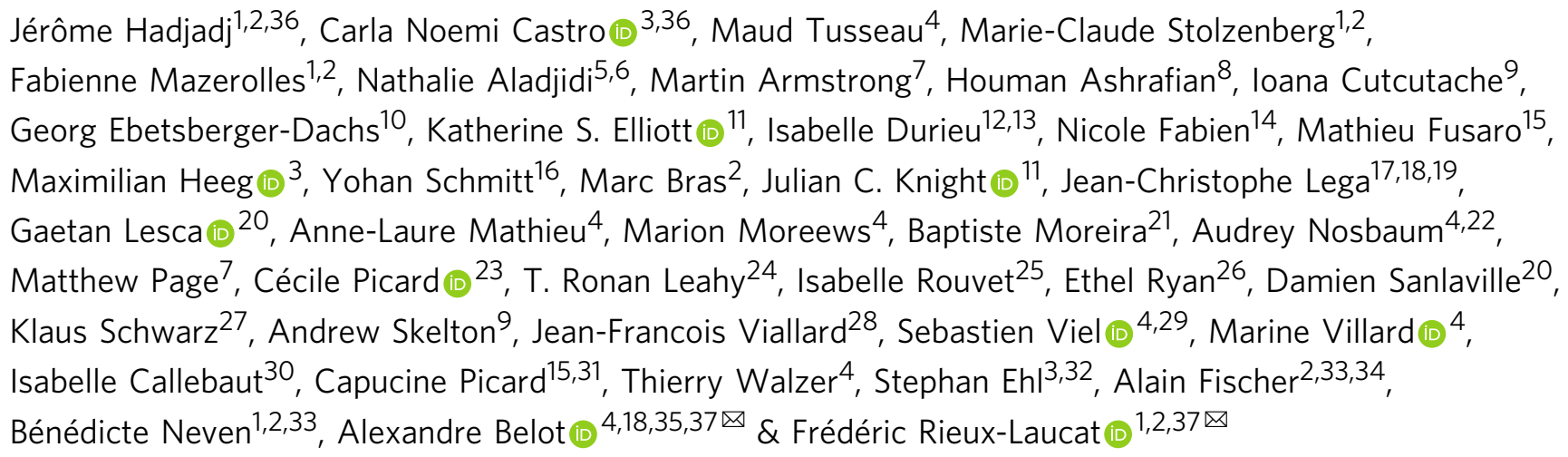

${ }^{1}$ Université de Paris, Imagine institute, laboratory of Immunogenetics of Pediatric Autoimmune Diseases, INSERM UMR 1163,24 boulevard du Montparnasse, 75015 Paris, France. ${ }^{2}$ Université de Paris, IHU-Imagine, 24 boulevard du Montparnasse, Paris 75015, France. ${ }^{3}$ Institute for Immunodeficiency, Center for Chronic Immunodeficiency, Medical Center - University of Freiburg, Faculty of Medicine, University of Freiburg, Freiburg, Germany. ${ }^{4}$ Centre International de Recherche en Infectiologie, CIRI, Inserm, U1111, Université Claude Bernard Lyon 1, CNRS, UMR5308, École Normale Supérieure de Lyon, University of Lyon, Lyon, France. ${ }^{5}$ Centre de Référence National des Cytopénies Auto-immunes de I'Enfant (CEREVANCE), CIC 1401, Inserm CICP, Bordeaux, France. ${ }^{\circ}$ Pediatric Oncology Hematology Unit, University Hospital, place Amélie Raba Léon, CIC 1401, Inserm, CICP, Bordeaux, France. ${ }^{7}$ Translational Medicine, UCB Pharma, Braine-l'Alleud, Belgium. ${ }^{8}$ Experimental Therapeutics, Radcliffe Department of Medicine, University of Oxford, Oxford, United Kingdom. ${ }^{9}$ Translational Medicine, UCB Pharma, Slough, United Kingdom.

${ }^{10}$ Department of Pediatrics, Kepler University Hospital and School of Medicine, Johannes Kepler University, Linz, Austria. ${ }^{11}$ Wellcome Centre for Human Genetics, University of Oxford, Oxford, UK. ${ }^{12}$ Internal Medicine and Vascular Pathology Department, Adult Cystic Fibrosis Center, Groupement Hospitalier Lyon-Sud, Hospices Civils de Lyon, Pierre-Bénite, France. ${ }^{13}$ EA 7425 HESPER. Université de Lyon, Lyon, France.

${ }^{14}$ Immunology laboratory; Centre Hospitalier Lyon Sud, Hospices Civils de Lyon, Lyon, France. ${ }^{15}$ Study Center for Primary Immunodeficiencies, APHP, Necker Hospital for Sick Children, Paris, France. ${ }^{16}$ Genomic Core Facility, INSERM UMR1163, Imagine Institute, Paris, France. ${ }^{17}$ Department of Internal and Vascular Medicine, Centre Hospitalier Lyon Sud, Hospices Civils de Lyon, Lyon, France. ${ }^{18}$ National Referee Centre for Pediatric-Onset Rheumatism and Autoimmune Diseases (RAISE), Lyon, France. ${ }^{19}$ UMR 5558, Equipe Evaluation et Modélisation des Effets Thérapeutiques, Laboratoire de Biométrie et Biologie Evolutive, CNRS, Claude Bernard University Lyon 1, Lyon, France. ${ }^{20}$ Service de Génétique, Hospices Civils de Lyon - GHE, and Institut Neuromyogène, CNRS UMR 5310 - INSERM U1217, Université de Lyon, Université Claude Bernard Lyon 1, Lyon, France. ${ }^{21}$ Immunology Laboratory, Necker Children's Hospital, Assistance Publique-Hôpitaux de Paris, Paris, France. ${ }^{22}$ Allergy and Clinical Immunology department, Groupement Hospitalier Lyon-Sud, Hospices Civils de Lyon, Pierre-Bénite, France. ${ }^{23}$ Institut de Pathologie Multisite, Groupement Hospitalier Est, Hospices Civils de Lyon, UCBL Lyon 1 University, Lyon, France. ${ }^{24}$ Department of Paediatric Immunology and Infectious Diseases, Children's Health Ireland at Crumlin, Dublin, Ireland. ${ }^{25}$ Centre de biotechnologie cellulaire et Biothèque, Groupe Hospitalier Est, Hospices Civils de Lyon, 69677 Bron, France. ${ }^{26}$ Department of Paediatrics, University Hospital Galway, Co, Galway, Ireland. ${ }^{27}$ Institute for Transfusion Medicin, University Ulm and Institute for Clinical Transfusion Medicine and Immunogenetics Ulm, German Red Cross Blood Service Baden-WürttembergHessen, 89081 Ulm, Germany. ${ }^{28}$ Département de Médecine Interne et Maladies Infectieuses, Centre Hospitalier Universitaire Haut Lévêque, Université de Bordeaux, Pessac, France. ${ }^{29}$ Service d'Immunologie Biologique, Groupement Hospitalier Lyon-Sud, Hospices Civils de Lyon, PierreBénite, France. ${ }^{30}$ Sorbonne Université, Muséum National d'Histoire Naturelle, Centre National de la Recherche Scientifique Unité Mixte de Recherche 7590, Institut de Minéralogie, de Physique des Matériaux et de Cosmochimie, Paris, France. ${ }^{31}$ Université de Paris, Imagine institute, laboratory of Iymphocyte activation and susceptibility to EBV, INSERM UMR 1163, 24 boulevard du Montparnasse, Paris 75015, France. ${ }^{32}$ CIBSS Centre for Integrative Biological Signalling Studies, University of Freiburg, Freiburg, Germany. ${ }^{33}$ Paediatric Immuno-Haematology and Rheumatology Department, Necker-Enfants Malades University Hospital, Assistance Publique-Hôpitaux de Paris, 75015 Paris, France. ${ }^{34}$ Collège de France, Paris, France. ${ }^{35}$ Hospices Civils de Lyon, Paediatric Nephrology, Rheumatology, Dermatology Unit, Mother and Children University Hospital, Bron, France. ${ }^{36}$ These authors contributed equally: Jérôme Hadjadj, Carla Castro. ${ }^{37}$ These authors jointly supervised this work: Alexandre Belot,

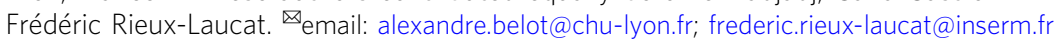

